\title{
Curative liver transplantation after lung resection for advanced hepato- cellular carcinoma with lung metastasis and inferior vena cava tumor thrombosis: a case report
}

\author{
Dong Jin Joo ${ }^{1,2}$, Do Young Kim³, Jinsil Seong ${ }^{4}$, Hyun Jeong Kim', Jae Geun Lee ${ }^{1,2}$, Dai Hoon Han', Gi Hong Choi', \\ Myoung Soo Kim ${ }^{1,2}$, Jin Sub Choi ${ }^{1,2}$, Soon Il Kim ${ }^{1,2}$ \\ ${ }^{1}$ Department of Surgery, ${ }^{2}$ The Research Institute for Transplantation, ${ }^{3}$ Department of Internal Medicine, ${ }^{4}$ Department of Radiation \\ Oncology, Yonsei Cancer Center, Yonsei University College of Medicine, Seoul, Korea
}

Received May 28, 2021

Revised Sep. 1, 2021

Accepted Sep. 8, 2021
Hepatocellular carcinoma $(\mathrm{HCC})$ with distant metastasis is an absolute contraindication for liver transplantation (LT). However, it is still unclear whether LT is feasible or acceptable in such patients, albeit after being treated with a multidisciplinary approach and after any metastatic lesion is ruled out. We report one such successful treatment with living donor LT (LDLT) after completely controlling far-advanced HCC with inferior vena cava tumor thrombosis and multiple lung metastases. The patient has been doing well without HCC recurrence for eight years since LDLT. The current patient could be an anecdotal case, but provides a case for expanding LDLT indications in the context of advanced HCC and suchlike. (J Liver Cancer 2021;21:181-186)

Keywords: Hepatocellular carcinoma; Neoplasm metastasis; Radiotherapy; Liver transplantation; Case report

\section{INTRODUCTION}

Because of the high risk of hepatocellular carcinoma (HCC) recurrence after liver transplantation (LT), there is a need for strict selection criteria for performing LT in order to achieve an optimal prognosis. Once HCC recurs after LT, the outcome of the patients is quite dismal and the five-year survival rates are expected to be less than 20\%. To avoid HCC recurrence after LT, careful patient selection is necessary. Conventionally, Milan criteria have been accepted as the gold

\section{Corresponding author: Dong Jin Joo}

Department of Surgery, Yonsei University College of Medicine, 50-1 Yonsei-ro, Seodaemun-gu, Seoul 03722, Korea Tel. +82-2-2228-2131, Fax. +82-2-313-8289 E-mail: djjoo@yuhs.ac standard to select LT for HCC, though several extended criteria such as University of San Francisco criteria, and up-toseven criteria exist. ${ }^{2}$ However, we still do not have definite criteria to decide on LT for advanced HCC patients. Milan criteria would be too limited to give a chance to those patients who could get more survival benefits from LT. For this reason, many centers have tried to expand their selection criteria. ${ }^{3}$

Despite these attempts, distant metastasis of HCC is considered to be an absolute contraindication for LT; LT cannot treat systemic metastasis and to date, there has been no report of LT in HCC patients with distant metastasis. Herein, we report a pioneering case of successful LT following multidisciplinary down-staging treatment for a patient with advanced HCC with inferior vena cava (IVC) tumor thrombo- 
sis and lung metastasis before transplantation. This case report is described according to the CARE guidelines available from https://www.care-statement.org/.

\section{CASE REPORT}

A 50-year-old male was referred to our center for the treatment of a large HCC in the right lobe of the liver in April 2009. Pain in the right upper quadrant started a month before he visited a local hospital. His condition was diagnosed with hepatitis B virus 25 years earlier. He had been adminis- tered lamivudine since 36 years of age; however, he quit lamivudine 3 years prior to the referral. He had no history of alcohol abuse but a smoking history of 30 pack-years. The patient's general condition was good. The laboratory findings were as follows: white blood cell count, $6,770 / \mu \mathrm{L}$; hemoglobin, $17.7 \mathrm{~g} / \mathrm{dL}$; platelets, $142,000 / \mu \mathrm{L}$; total bilirubin, $1.0 \mathrm{mg} / \mathrm{dL}$; albumin, $4.1 \mathrm{~g} / \mathrm{dL}$; international normalized ratio, 1.22; aspartate aminotransferase, $94 \mathrm{IU} / \mathrm{L}$; alanine aminotransferase, $65 \mathrm{IU} / \mathrm{L}$; blood urea nitrogen, $12.1 \mathrm{mg} / \mathrm{dL}$; and creatinine, $0.92 \mathrm{mg} / \mathrm{dL}$. The alpha-fetoprotein (AFP) level was $9.62 \mathrm{ng} / \mathrm{mL}$, and protein induced by vitamin $\mathrm{K}$ absence
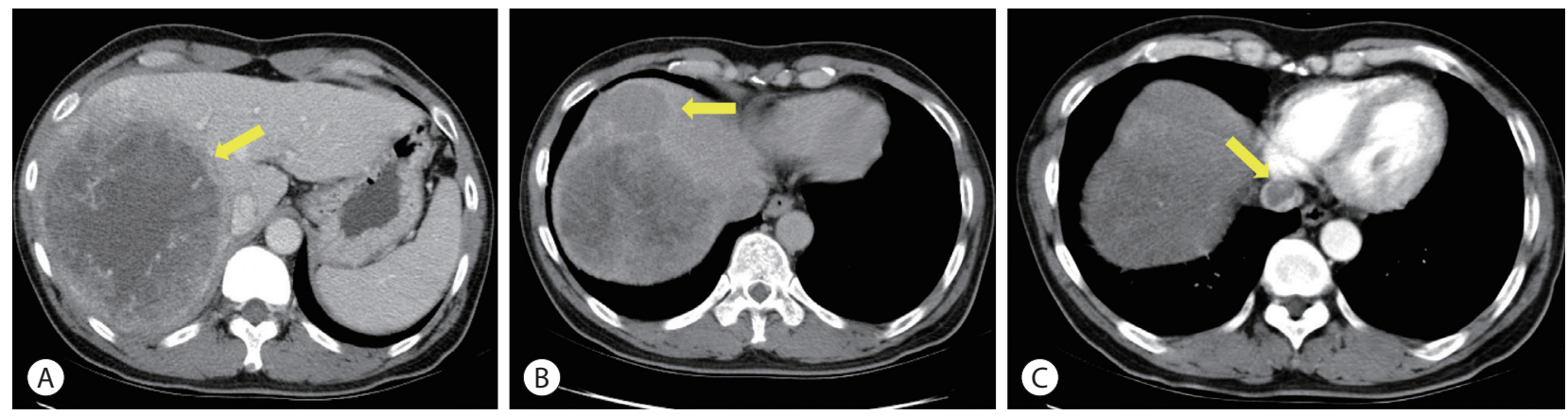

Figure 1. Initial computed tomography scan. A large hepatocellular carcinoma entirely occupied the right lobe (A), multiple satellite nodules (B) are seen. Tumor thrombosis is seen in the suprahepatic inferior vena cava (C) (arrows).
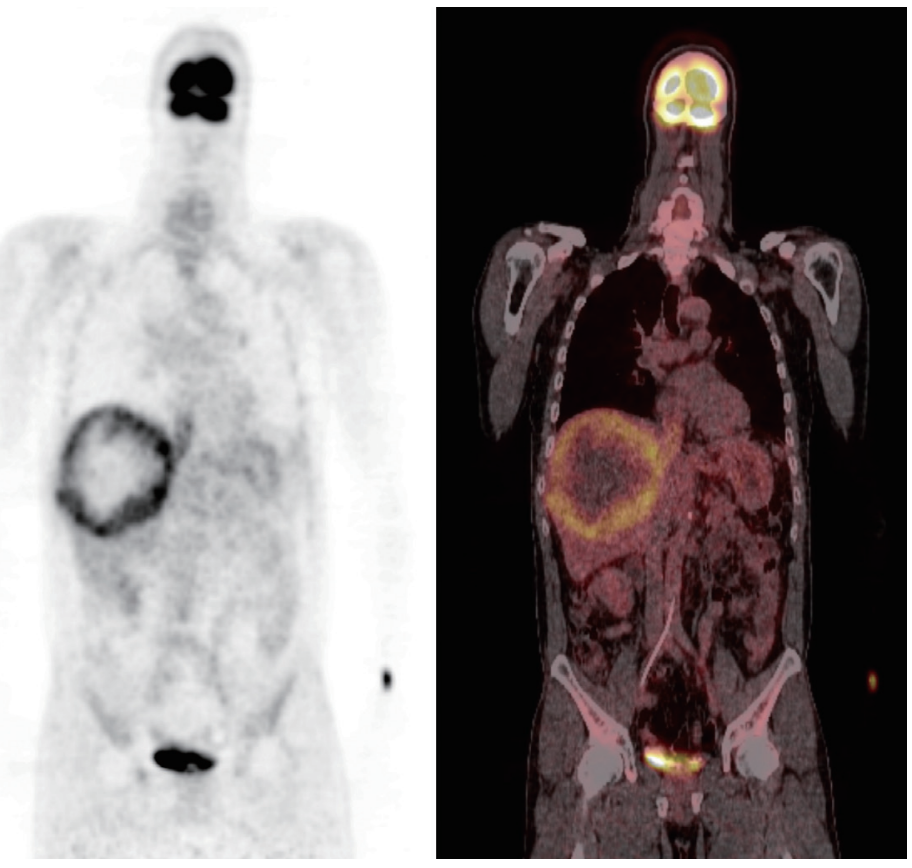

Figure 2. Initial positron emission tomography computed tomography scan. A large mass with increased fluorodeoxyglucose (FDG) uptake in the right lobe of the liver indicated high-grade hepatocellular carcinoma. Tumor thrombosis in the inferior vena cava shows FDG uptake. 
or antagonist-II (PIVKA-II) level was $>2,000$ pg/mL. Liver function was preserved (Child-Pugh score, A). Serological tests for hepatitis B surface antigen and antibody to the core antigen were positive, but were negative for the antibody against hepatitis $\mathrm{C}$ virus.

Initial contrast-enhanced computed tomography (CT) showed a large hypervascular mass in the right lobe with hepatic veins, IVC thrombosis, and multiple satellite nodules (Fig. 1). Positron emission tomography (PET)-CT showed a large mass with increased fluorodeoxyglucose (FDG) uptake in the right lobe of the liver, indicating high-grade HCC. Tumor thrombosis with FDG uptake in the IVC was observed (Fig. 2). There was bilateral pulmonary arterial thromboembolism with FDG uptake, which resulted in possible tumor thrombi. It revealed multifocal consolidations and ill-defined nodules in both lungs, which indicated infarction due to embolism.

Concurrent chemoradiotherapy (CCRT) followed by hepatic artery infusion chemotherapy (HAIC) was chosen as the first treatment after talks in a multidisciplinary conference. ${ }^{4}$ Three-dimensional conformal radiotherapy was performed with 45 Gy in 25 fractions for 5 weeks. After CCRT, the primary tumor decreased and was near-totally necrotized. However, in the CT scan taken for response evaluation, there were newly developed multiple lung metastases, even though the primary liver tumors showed partial response to treatment (Fig. 3). We continued HAIC with 5-fluorouracil (at a dose of $500 \mathrm{mg} / \mathrm{m}^{2}$ for 5 hours on days 1 to 3 ) and cisplatin (at $60 \mathrm{mg} / \mathrm{m}^{2}$ for 2 hours on day 2). After 15 cycles of HAIC treatment, all hematogenous lung metastatic lesions, except a single lesion on the right upper lung, disappeared. The residual single metastatic nodule showed an increased size (Fig. 4A). At the multidisciplinary conference, we decided to perform lung wedge resection to remove residual nodules in the lung. Video-assisted thoracoscopic (VATS) wedge resection was performed. The histological findings of the lesion showed poorly differentiated carcinoma, favoring metastatic HCC (Fig. 4B).

Even after lung resection, HCC recurred repeatedly in other parts of the liver. We performed radiofrequency ablation (RFA) for a $2.6 \mathrm{~cm}$-sized tumor on segment 2, 1 month after VATS. Transarterial chemoembolization (TACE) was performed for three recurrent lesions on segment 6, 6 months after the RFA. Finally, another RFA treatment was applied to
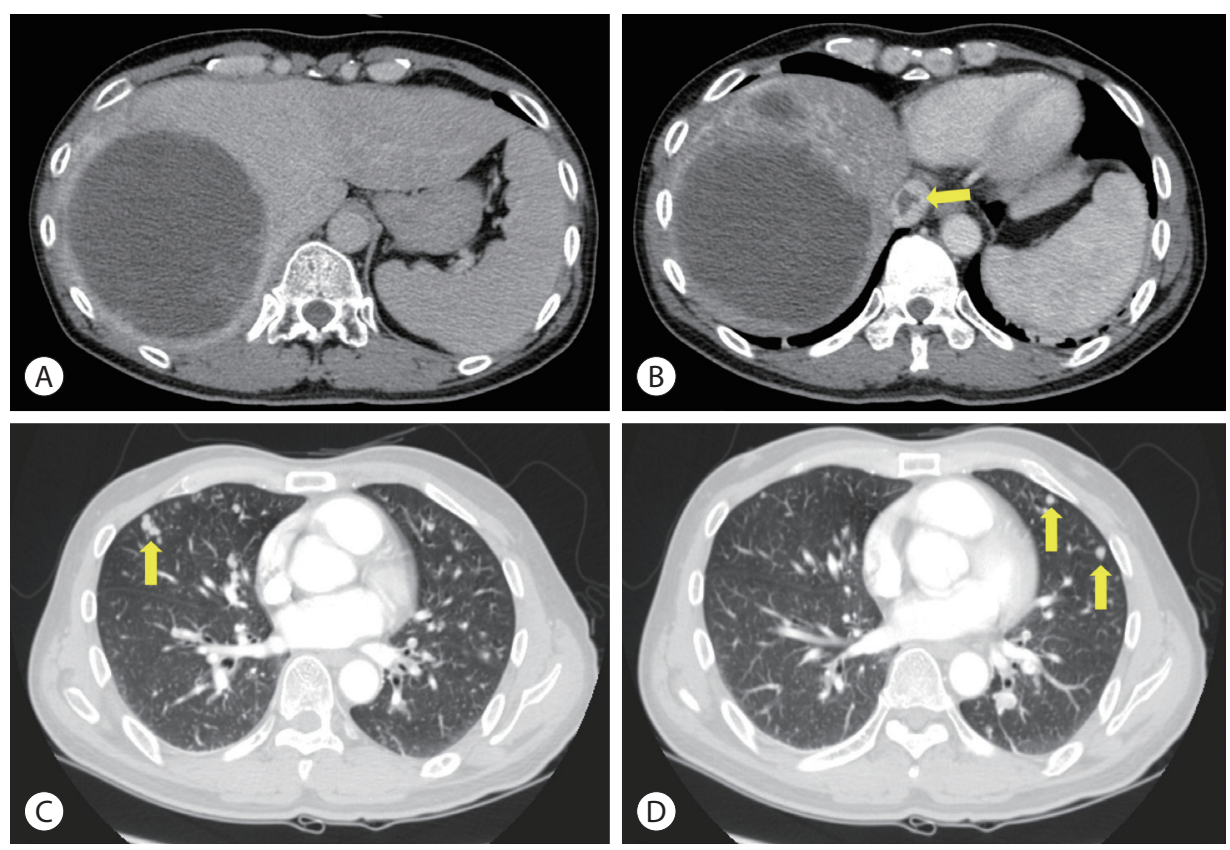

Figure 3. Liver and chest computed tomography scan after concurrent chemoradiotherapy. The primary tumor decreases and is near-totally necrotized (A). Inferior vena cava thrombosis reduces (B). However, multiple metastatic nodules are seen in both lung fields (C, D) (arrows). 
segment 2, 10 months after TACE.

After completing the above treatments for recurrent lesions in the liver, there were no more viable tumors in imaging studies, including CT and PET-CT scans. However, liver function of the patient gradually worsened. His platelet count decreased to $22,000 / \mu \mathrm{L}$, and his total bilirubin level was elevated to $2.6 \mathrm{mg} / \mathrm{dL}$. After extensive internal discussions on whether LT could be beneficial in this scenario, we decided to wait for more than 3 months to define the tumor characteristics before progressing with LT. The patient and his family members were very favorable toward LT and were willing to take the risk of HCC recurrence after LT. Meanwhile, we evaluated his son as a live donor. As there was no recurrence during the waiting period, we planned a living donor LT (LDLT). This LDLT procedure was accomplished 4 months after the last locoregional treatment, which was almost 4 years after the initial diagnosis.

The patient underwent LDLT with a modified right lobe graft in January 2013. At the time of LDLT, his AFP and PIVKA-II tumor markers were $65.68 \mathrm{ng} / \mathrm{mL}$ in AFP and $32 \mathrm{mAU} / \mathrm{mL}$, respectively. He recovered uneventfully with standard postoperative care and immunosuppression. The explanted liver showed four HCCs that were near-totally necrotized, with no microvascular invasion (Fig. 5).
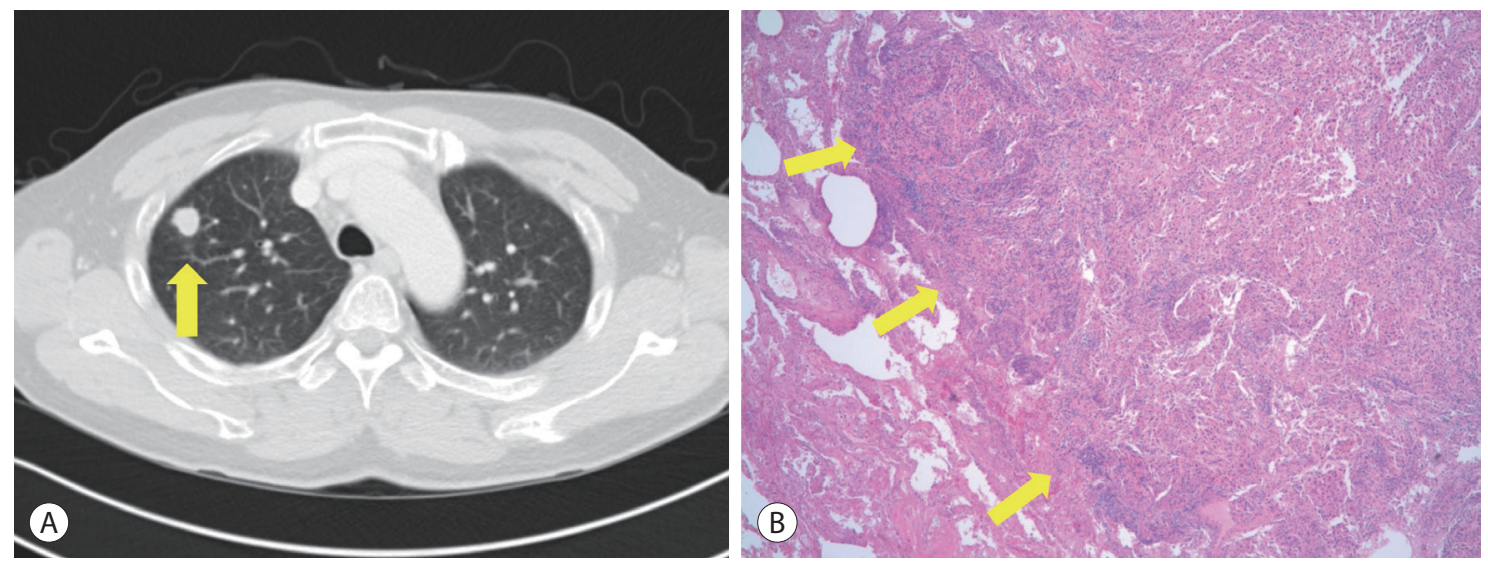

Figure 4. Chest computed tomography scan after 15 cycles of hepatic artery infusion chemotherapy. All the hematogenous lung metastatic lesions except a single lesion on the right upper lung disappear. The residual single metastatic nodule shows increased size ( $A$, arrow). The histological findings show poorly differentiated carcinoma, favoring metastatic hepatocellular carcinoma ( $B$, arrows indicate the tumor margin) (hematoxylin and eosin, $\times 40$ ).
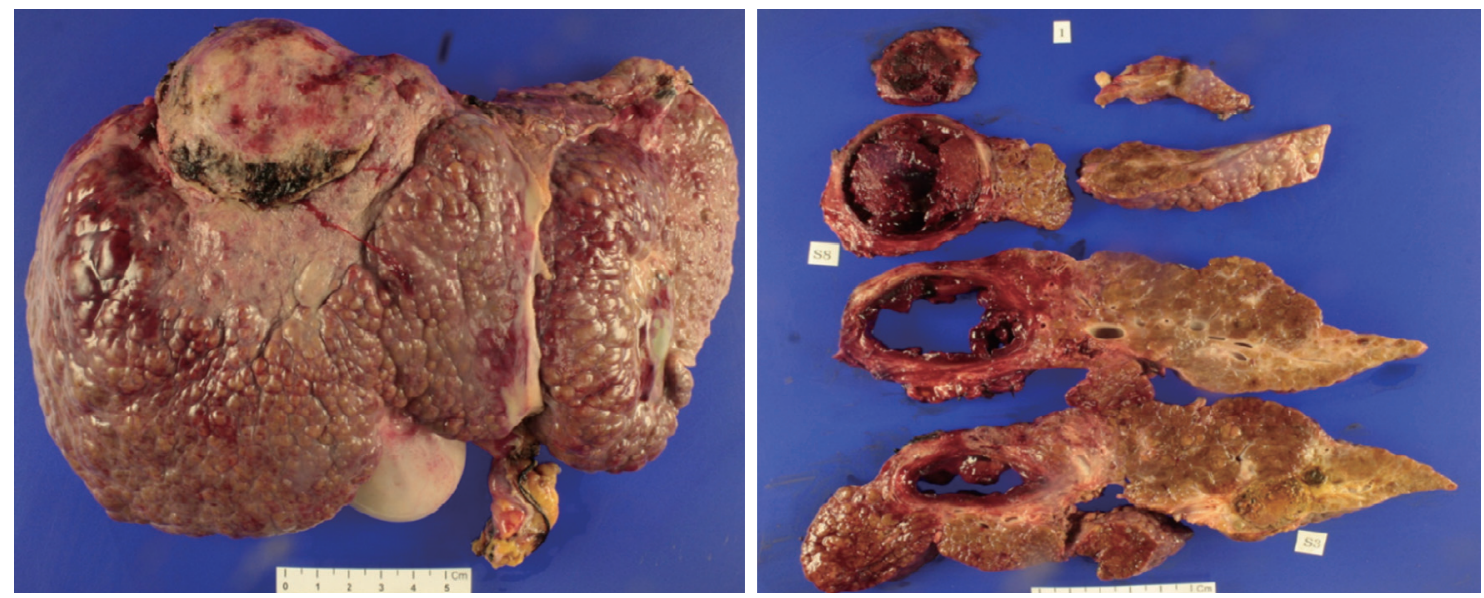

Figure 5. Explanted liver. The explanted liver shows four hepatocellular carcinoma tumors that were totally necrotized with no microvascular invasion. The largest tumor is $6 \times 5.5 \mathrm{~cm}$ in size (on segment 5); there are no viable lesions. 
We started his maintenance immunosuppression with tacrolimus and low-dose steroid and substituted tacrolimus with the mammarian target of rapamycin (mTOR) inhibitor, sirolimus 3 months after LT. Since then, he has been administered sirolimus monotherapy for immunosuppression without calcineurin inhibitors and steroids. It has been 8 years since the patient underwent LDLT. There has been no HCC recurrence so far. He is doing well, without experiencing any complications related to LT or immunosuppression.

\section{DISCUSSION}

As HCC primarily arises from chronic liver disease, LT replacing the native liver has been established as the standard treatment in selected patients for the last two decades. ${ }^{5}$ However, applying LT to far-advanced HCC is still controversial because the risk of recurrence of these tumors is very high, and a good result may not be expected. However, many centers have attempted to expand the selection criteria for HCC patients in order for them to undergo LT. It seems that the Milan criteria would not be the Bible anymore. ${ }^{3,6-8}$ Nevertheless, distant metastasis of HCC is still considered an absolute contraindication for LT. There is no literature on successful LT performed on a patient with metastatic HCC. Since 2012, our center has been trying to expand LT criteria for advanced HCC through a multidisciplinary approach. When the down-staging for those patients succeeds, we carefully select them to undergo LT. We reported an acceptable result of our initial experience with curative LT for patients who had portal vein tumor thrombosis after successful down-staging with CCRT. ${ }^{9}$ In this study, we reported that five patients out of eight had reached the 3-year mark of recurrence-free survival even though they were considered as a contraindication for LT, and their life expectancy would have been less than 1 year without LT. From this early experience, we learned that careful selection could show good outcomes even for patients with far-advanced HCC with portal vein tumor thrombosis who showed good response to pretransplant locoregional treatment, including CCRT. We selected one such patient, carefully considering the tumor biology and observ- ing the disease progress. We discussed all possible outcomes with his family members, and obtained informed consent. All family members were eager for LT, and they were willing to donate their liver to the patient. Due to organ shortage problem, to avoid futile transplantation, strict selection criteria for patients with HCC have been considered in deceased donor liver transplants. But, LDLT has different story because the graft should be used only for the family member. Thus, we could cautiously expand LT criteria for those with advanced HCC to include those who did not show any disease progression after down-staging therapy as long as the patient and family members understand all the circumstances and are willing to risk HCC recurrence in the absence of other options. Disease progression even after locoregional or systemic therapy before LT can be one of the independent risk factors for HCC recurrence after LT, implying aggressive tumor characteristics. ${ }^{10}$ Thus, we waited at least 3 months after the last RFA to perform LT and checked if there was no viable tumor in the liver and lung just before the transplantation. This patient had not shown any disease progression for over 3 months.

As HCC treatment should vary according to tumor size, number, location, and aggressiveness, a multidisciplinary approach is necessary to determine treatment modalities. Our center has regularly discussed complicated HCC cases at multidisciplinary conferences that include hepatologists, radiation oncologists, liver surgeons, medical oncologists, interventional radiologists, and nuclear medicine specialists. This led us to have established our unique treatment for advanced HCC, similar to the current case, which is CCRT followed by HAIC. ${ }^{4}$ Utilizing this protocol, we have shown promising results, making the unresectable or non-transplantable patients undergo curative surgery such as liver resection or transplantation. ${ }^{9,11}$ We believe the patient, in this case, gained the absolute benefit of multidisciplinary treatment. He was a good responder to CCRT and systemic chemotherapy, which made him undergo curative surgery.

In conclusion, we cannot generalize this case for all patients with distant metastasis of HCC. This patient might have been an anecdotal case. However, this report suggests that metastatic HCC should not be an absolute contraindica- 
tion if the patient is a good responder to combined locoregional and systemic therapy, and if there is no more viable lesion at the time of the LT procedure.

\section{Conflicts of Interest}

The authors have no conflicts of interest to disclose.

\section{Ethics Statement}

This case report was approved by the Ethical Committee and Institutional Review Board of Severance Hospital (IRB No.4-2021-1001). The operation was performed after obtaining informed consent from the patient and his family members.

\section{Funding Statement}

No funding to declare.

\section{Data Availability}

Data sharing not applicable to this article as no datasets were generated or analyzed for this case report.

\section{ORCID}

$$
\begin{array}{ll}
\text { Dong Jin Joo } & \text { https://orcid.org/0000-0001-8405-1531 } \\
\text { Do Young Kim } & \text { https://orcid.org/0000-0002-8327-3439 } \\
\text { Jinsil Seong } & \text { https://orcid.org/0000-0003-1794-5951 } \\
\text { Hyun Jeong Kim } & \text { https://orcid.org/0000-0002-6033-5094 } \\
\text { Jae Geun Lee } & \text { https://orcid.org/0000-0002-6722-0257 } \\
\text { Dai Hoon Han } & \text { https://orcid.org/0000-0003-2787-7876 } \\
\text { Gi Hong Choi } & \text { https://orcid.org/0000-0002-1593-3773 } \\
\text { Myoung Soo Kim } & \text { https://orcid.org/0000-0002-8975-8381 } \\
\text { Jin Sub Choi } & \text { https://orcid.org/0000-0002-6467-6494 } \\
\text { Soon Il Kim } & \text { https://orcid.org/0000-0002-0783-7538 }
\end{array}
$$

\section{Author Contribution}

Conceptualization: DJJ

Data curation: DJJ, HJK

Methodology: DYK, JS, JGL

Project administration: DJJ, JGL
Writing-original draft: DJJ

Writing-review \& editing: DJJ, DYK, JS, JGL, DHH, MSK,

$$
\text { HJK, JSC, SIK }
$$

Approval of final manuscript: all authors

\section{References}

1. Kaido T, Mori A, Ogura Y, Hata K, Yoshizawa A, lida T, et al. Recurrence of hepatocellular carcinoma after living donor liver transplantation: what is the current optimal approach to prevent recurrence? World J Surg 2011;35:1355-1359.

2. Mazzaferro V, Bhoori S, Sposito C, Bongini M, Langer M, Miceli R, et al. Milan criteria in liver transplantation for hepatocellular carcinoma: an evidence-based analysis of 15 years of experience. Liver Transpl 2011;17 Suppl 2:S44-57.

3. Lingiah VA, Niazi M, Olivo R, Paterno F, Guarrera JV, Pyrsopoulos NT. Liver transplantation beyond Milan criteria. J Clin Transl Hepatol 2020;8:69-75.

4. Han KH, Seong J, Kim JK, Ahn SH, Lee DY, Chon CY. Pilot clinical trial of localized concurrent chemoradiation therapy for locally advanced hepatocellular carcinoma with portal vein thrombosis. Cancer 2008;113:995-1003.

5. Elshamy M, Aucejo F, Menon KV, Eghtesad B. Hepatocellular carcinoma beyond Milan criteria: Management and transplant selection criteria. World J Hepatol 2016;8:874-880.

6. Yoshizumi T, Shirabe K, Mori M. It is time to abandon the Milan criteria. Hepatobiliary Surg Nutr 2019;8:56-58.

7. Ho CM, Hu RH. Comment on "Is it time to abandon the Milan criteria?". Ann Surg 2019;270:e85.

8. Xu X, Lu D, Ling Q, Wei X, Wu J, Zhou L, et al. Liver transplantation for hepatocellular carcinoma beyond the Milan criteria. Gut 2016;65:1035-1041.

9. Han DH, Joo DJ, Kim MS, Choi GH, Choi JS, Park YN, et al. Living donor liver transplantation for advanced hepatocellular carcinoma with portal vein tumor thrombosis after concurrent chemoradiation therapy. Yonsei Med J 2016;57:1276-1281.

10. De Carlis L, Di Sandro S, Giacomoni A, Slim A, Lauterio A, Mangoni I, et al. Beyond the Milan criteria: what risks for patients with hepatocellular carcinoma progression before liver transplantation? J Clin Gastroenterol 2012;46:78-86.

11. Lee WH, Byun HK, Choi JS, Choi GH, Han DH, Joo DJ, et al. Liverdirected combined radiotherapy as a bridge to curative surgery in locally advanced hepatocellular carcinoma beyond the Milan criteria. Radiother Oncol 2020;152:1-7. 